\title{
Negative thermal bond expansion of a molecular solid, $\mathrm{Tc}_{2} \mathrm{O}_{7}$
}

Daniel Mast, ${ }^{1,2,3}$ Bradley Childs, ${ }^{1,2}$ Keith V. Lawler, ${ }^{1,3}$ Frederic Poineau, ${ }^{1,3}$ Alfred P. Sattelberger ${ }^{4}$, Kenneth R. Czerwinski ${ }^{1,2}$ and Paul M. Forster, ${ }^{1,2,3}$

${ }^{1}$ Department of Chemistry and Biochemistry, ${ }^{2}$ Radiochemistry Program, ${ }^{3}$ High Pressure Science and Engineering Center, University of Nevada Las Vegas, Las Vegas, NV 89154, USA

${ }^{4}$ Energy Engineering and Systems Analysis Directorate, Argonne National Laboratory, Argonne, IL 60439, USA

The binary oxides of group 7 transition metal form a series of unique structures. Two of the three crystallize as molecular solids with two metal centers bridged by a single oxygen. Molecular metal oxides stable under ambient conditions are rare and offer unique insight into the chemistry and physics of materials. The structure of $\mathrm{Tc}_{2} \mathrm{O}_{7}$ is characterized by centrosymmetric linear molecules with a single bridging oxygen. In a combined computational and experimental investigation, anomalous thermal expansion behavior has been identified between room temperature and $100 \mathrm{~K}$. Using variable temperature single crystal diffraction and Langevin Molecular dynamics we can model the negative thermal bond expansion. 